\title{
Expression Pattern of Hyaluronan Synthase2 in Inflammatory Breast Cancer Cells N.M.Mohamed ${ }^{1}$, M.S.Zaghloul ${ }^{1}$, O.I.Ghonemy ${ }^{1}$, M.El-Shinawi ${ }^{2}$ and S.A.Ibrahim ${ }^{3}$ \\ ${ }^{1}$ Zoology Dept., Faculty of Science, Benha Univ., Benha, Egypt \\ ${ }^{2}$ General Surgery Dept., Faculty of Medicine, Ain Shams Univ., Cairo, Egypt \\ ${ }^{3}$ Zoology Dept, Faculty of Science, Cairo Univ., Giza, Egypt. \\ E-Mail: noura.nabawy@fsc.bu.edu.eg
}

\begin{abstract}
Breast cancer (BC) Is the most widely recognized threatening malignant disease around the whole world. It Is the second most incessant reason for malignant tumor growth related to death among woman at different ages. Inflammatory Breast Cancer (IBC) Is the most lethal form of BC with low survival rate. Because of lacking molecular markers, identification of new unique markers for IBC Is warranted. Hyaluronic acid synthases (HASs), particularly HAS2, have shown a critical role in the metastasis of non-inflammatory breast cancer (nonIBC). In this study, we assessed HAS2 mRNA expression by qPCR in the human triple negative non-IBC MDAMB-231 and IBC SUM-149 cell lines. Our data demonstrated that HAS2 mRNA expression was significantly upregulated in IBC vs. non-IBC breast cancer cell lines. In conclusion, the high expression of HAS2 may represents a marker for IBC and could have a role in IBC pathogenesis.
\end{abstract}

Keywords: HAS2, Breast cancer, Inflammatory breast cancer.

\section{Introduction}

Inflammatory breast cancer (IBC), the most aggressive type of breast cancer, with a ratio that up to $2.5 \%$ of newly diagnosed breast cancers in the United States[1]. This ratio arrives at a considerably more significant level of 5-10\% of breast cancer cases in North African countries like Tunisia, Morocco, and Egypt[2]. IBC Is a unique disease with distinctive Physical Properties such as; reddening of the skin (erythema), the breast becomes larger in size due to edema which accompanies overstated hair- follicle bores which makes the skin of the breast appears as an "Orange Peel" and presences of lymphatic tumor emboli[3]. IBC patients have low odds of survival rate with an average of 3 years when compared to non-IBC besides the current lack of the appropriate treatment methods [1]. In vertebrates, and comparing to other GAGs, (HA) Is the largest polysaccharide Is found in extra-cellular matrix [4],[5],[36], [38], [39]. Simply with a linear, non-sulfated, repeated and unbranched disaccharides forming finally polysaccharide (glycosaminoglycan) that Is made of D-glucuronic acid and N-acetyl-D-glucosamine as substrates [4],[36], [38], [39]. HA Is with a key role in many of Physiological and Pathological processes including tissue development, inflammation, wound healing, tumor Progression and metastasis including adhesion and migration[6]-[8]. Besides its importance in other processes as Proliferation, and apoptosis[9]-[13]. It was demonstrated that HA Is responsible for tumor cell migration and invasion in vitro, and regulates tumor growth and Progression in vivo[14]. Number of cell culture studies demonstrated that HA accumulates in larger amount in invasive breast cancer cells than both normal tissues and less aggressive cancerous cells, especially HA that Is synthesized by HAS2 [15].

There are three hyaluronan synthase isoforms, known as HAS1, HAS2 and HAS3, and they all can produce HA at the inner side of the cytoplasmic membrane in vertebrates[16],[17]. They can produce different sizes of HA molecules with somehow different functions. HAS1 and HAS2 produce the large size of HA whereas HAS3 produces HA of a smaller molecular mass [18]. HAS2 knockout mice die at embryonic day9.5 due to cardiovascular defects [8], [19]-[21]. Which makes HAS2 the major isoform responsible for the synthesis of hyaluronan in mesenchymal cells[19]. That make us believe that it may have a critical role in IBC Progression and subsequent metastasis. So, the aim of this study was to characterize the expression of HAS enzymes in IBC vs non-IBC human breast cancer cell lines on mRNA level using real-time PCR (qPCR).

\section{Material and methods \\ 2.1Cell lines}

In this study, we used two human breast cancer cell lines MDA-MB-231 (ATCC® HTB-26 ${ }^{\mathrm{TM}}$ ), representing non-IBC, and SUM-149 (a kindly gift from Bonnie Sloan, Wayne State University, Detroit, MI, USA) representing IBC. MDA-MB-231 cells were cultured in DMEM medium with $10 \%$ fetal bovine serum and $1 \%$ of Penicillin/streptomycin antibiotic mixture. SUM-149 cells were cultured in HAM's F12 medium with $5 \%$ fetal bovine serum, $1 \mu \mathrm{g} / \mathrm{mL}$ hydrocortisone, $5 \mu \mathrm{g} / \mathrm{mL}$ insulin and $1 \%$ of Penicillin/streptomycin antibiotic mixture. Both cell lines were incubated at $37^{\circ} \mathrm{C}$ in 5\% CO2 [22].

\subsection{Quantitative real time PCR}

Total cellular RNA was isolated from tissues using Trizol (invitrogen) and $1 \mu \mathrm{g}$ RNA was reverse transcribed into cDNA Thermo Fisher Scientific RevertAid First Strand cDNA Synthesis Kit (Thermo Fisher Scientific, CA, USA). Quantitative real-time PCR will be conducted in duplicate for each gene of 
interest using SYBR Green dye and gene expression levels was measured in a StePOnePlus detection System (Applied Biosystems, CA, USA). The $\Delta \mathrm{Ct}$ method was used to determine relative gene transcript levels of HAS enzymes after normalization to 18S rRNA as previously described (Ibrahim et al., 2017). Each sample was initially denatured at $95^{\circ} \mathrm{C}$ for $10 \mathrm{~min}$, then subjected to 40 cycles of the following: Denaturation at $95^{\circ} \mathrm{C}$ for 15 sec, annealing at $55^{\circ} \mathrm{C}$ for $30 \mathrm{sec}$ and extension at $60^{\circ} \mathrm{C}$ for $30 \mathrm{~min}$. Relative gene expression was evaluated using the $(2-\Delta \Delta \mathrm{Ct})$ method after normalization to $18 \mathrm{~S}$ rRNA. HAS2 and 18S Primers were purchased from Qiagen (Hilden, Germany).

\section{Statistical Analysis}

All data were analyzed by using Student's t-test (for normally distributed data) or Mann-Whitney U-test (for non- normally distributed data) was used for two group comparisons. Unless otherwise stated, all data are expressed as mean \pm SEM. The level of significance was set at $\mathrm{P}<0.05$.

\section{Results and discussion}

A higher Expression of HAS2 mRNA in SUM149 than that in MDA-MB-231 cells

The mRNA expression level of HAS2 IBC $(n=3)$ vs. non-IBC $(\mathrm{n}=3)$ human breast cancer cell lines was assessed using qRT-PCR. We found that there was a significant $(\mathrm{P}=0.04)$ increase of HAS2 mRNA expression in SUM-149 in comparison to MDA-MB231 cells Fig (1) and Fig ( $2 a \& 2 b)$.

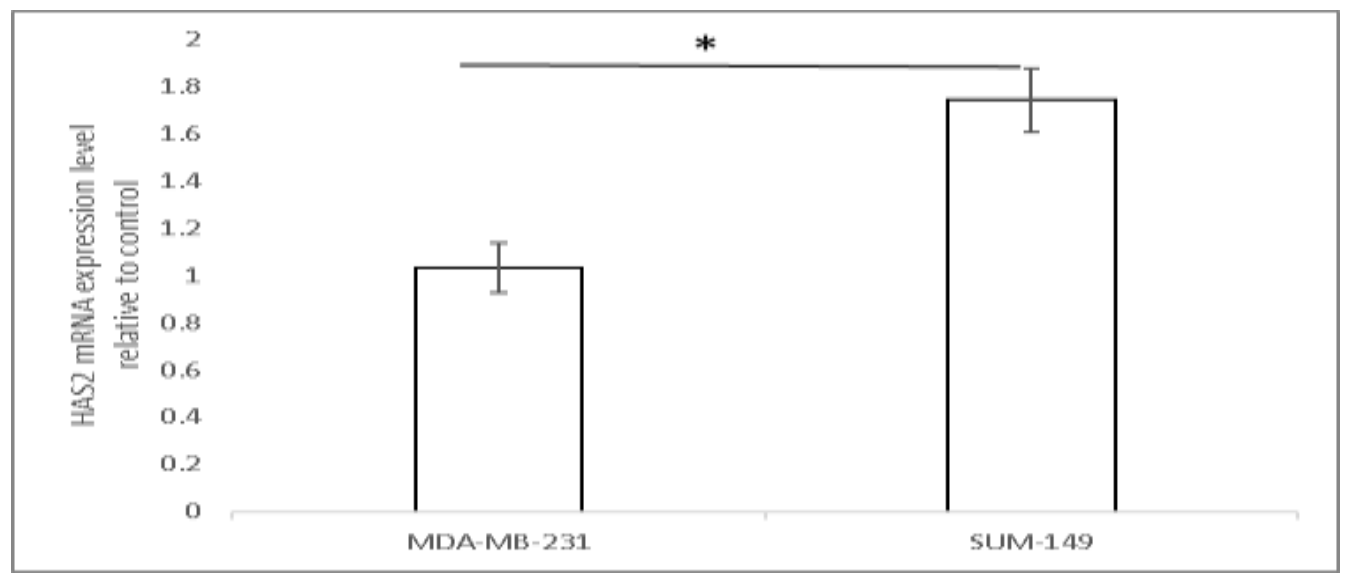

Fig(1) Expression of HAS2 mRNA in human breast cancer cell lines of IBC vs. non-IBC by using qPCR. The expression of HAS2 was upregulated in IBC $(n=3)$ relative to non-IBC $(n=3)$. $* \mathrm{P}<0.04$ as determined by Student's t-test.

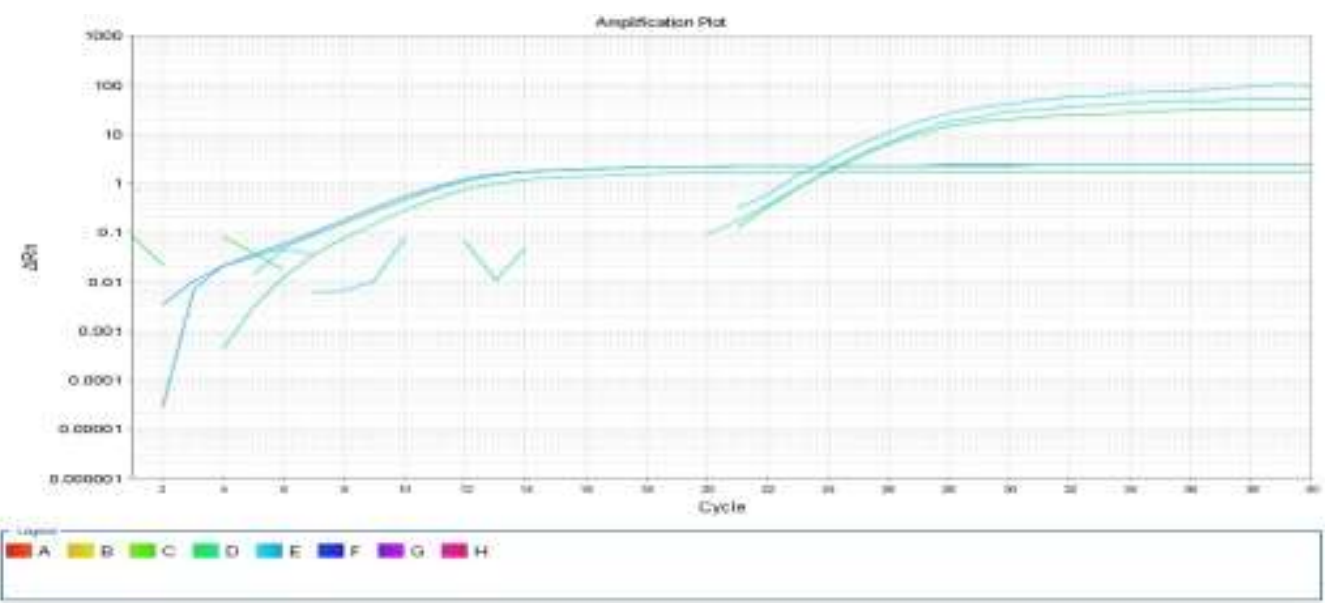

Fig (2a) Representative graph of the raw data analysis shows HAS2 and 18S amplification Plots of MDA-MB-231 cell lines $(n=3)$. 


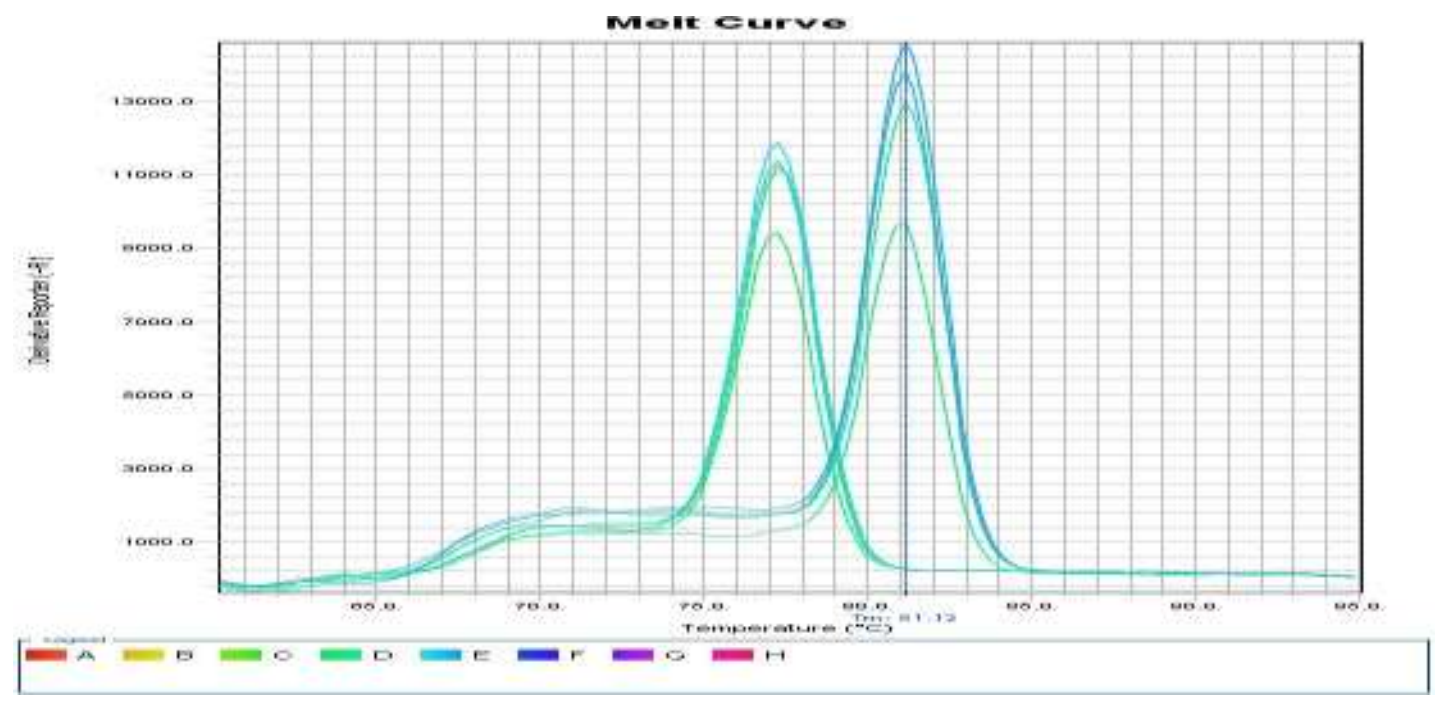

Fig(2b) Representative graph of the raw data analysis shows HAS2 and 18S melt curves of MDA-MB-231 cell lines $(n=3)$.

In many cancers, high levels of HA indicate an aggressive Progression and less survival rates as in colorectal, breast, Prostate, lung, ovarian, and gastric tumors [23]. Further studies were focused on HA and breast cancer (BC) showed a tight connection between HA levels and the aggressiveness of (BC); a HA-rich microenvironment induces tumors progress more aggressively as stromal HA makes both host and tumor cells to accelerate Progression[14], [24]. HA has been reported as an important key that roles the Proliferation, Progression and invasion of breast cancer cells, as high expression levels of HAS2 and its subsequent production of HA was demonstrated in invasive breast cancer cell lines such as MDA-MB-231 cells[25]. A comparison was made between invasive MDA- MB231-BM and MDA- MB-231 cells showed that the former produce high amounts of hyaluronan through over-production of the HAS2 gene than the letter ones[26]. Moreover, the overproduced HA especially by HAS2 transgene, caused a rapid growth and development of carcinoma using a mouse model for breast cancer[27]. Likewise, the triple-negative MDAMB-231 and HS578T cells have shown a higher expression levels of HAS2 mRNA and thus, produce higher amounts of HA than breast cancer cell lines with a less aggressive phenotype, like MCF-7 [28]. Li and colleagues, 2015, showed that HAS2 was up-regulated in breast cancer cell lines and invasive duct cancer tissues compared with normal breast tissues when they induced the expression of HAS2 transcripts and protein in breast cancer cell lines such as MCF-7, MDA-MB231, nonmalignant breast cell lines MCF- 10A and HBL-100, 55 invasive ductal carcinoma breast tissues and 55 relative normal breast tissues [24].
Also, the upregulation of HAS2 has been connected to the development of tumorigenesis causing both, cancer invasiveness and metastasis[29]. Higher expression of HAS2 was associated with an EpithelialMesenchymal Transition (EMT) phenotype in more than $70 \%$ of metaplastic breast carcinoma[30]. Furthermore, it was reported that the expression of HAS2 in cancer associated fibroblasts (CAFs), has a key role in stimulating the invasion of oral cancer cells [31]. It was also found that, HAS2 promotes pancreatic cancer cell motility. Besides, its aggregates was found in patients with malignant mesotheliomas within their pleural fluids and ascites [32]. Furthermore, HAS2 expression Is significantly upregulated in bone metastases in contrast to the original MDA-MB-231 cells, which means that HAS2 expression Is crucial for cell motility and invasion [33]. Likewise, biopsies that were taken from mammary tumor that has upregulated HAS2 expression promoted angiogenesis and inflammatory cells induction. For further confirmation of HAS2 role in cancer, it was knockout using RNA antisense or HA-synthesis inhibition by 4-MU, which led to a reduction of tumorigenesis and progression of invasive breast cancer cells [25]. Li and colleagues, 2007, showed that silencing of HAS2 in Hs578T breast cancer cells reduces their motility and invasive nearly to half of that cells express HAS2[15]. Actually, HA was noted in high levels around tumor cells or within the tumor stroma, in Patients with breast and ovarian carcinomas, high levels of HA were demonstrated in the stroma and were connected to low survival rates[10]. 


\section{Conclusion}

In this study, we demonstrated higher expression levels of HAS2 mRNA level in the human IBC SUM149 relative to MDA-MB-231 cells. Overall, we suggest that the high expression of HAS2 may act as a biomarker for IBC, and its targeting may represent a therapeutic strategy.

\section{References}

[1] K. W. Hance et al., "Trends in inflammatory breast carcinoma incidence and survival: The surveillance, epidemiology, and end results program at the National Cancer Institute," J. Natl. Cancer Inst., VOL. 97 (13), PP. 966-975, 2005.

[2] A.S. Soliman et al., "High Proportion of Inflammatory Breast Cancer in the PopulationBased Cancer Registry of Gharbiah, Egypt," Bone, VOL. 23 (1), PP. 1-7, 2011.

[3] G. Somlo and V. Jones, "Inflammatory breast cancer," Breast Compr. Manag. Benign Malig. Dis., VOL. 60 (6), PP. 832-838.e2, 2017.

[4] D. Chester and A. C. Brown, "The role of biophysical properties of provisional matrix proteins in wound repair," Matrix Biol., 2016.

[5] K. L. Perkins et al., "Brain extracellular space, hyaluronan, and the prevention of epileptic seizures," VOL. 28 (8), PP. 869-892, 2017.

[6] S. Adamia et al., "Hyaluronan and hyaluronan synthases: Potential therapeutic targets in cancer," Curr. Drug Targets - Cardiovasc. Haematol. Disord., VOL. 5 (1), PP. 3-14, 2005.

[7] K. T. Dicker et al., "Hyaluronan: A simple polysaccharide with diverse biological functions," Acta Biomater., VOL. 10(4), PP. 1558-1570, 2014.

[8] R. H. Tammi et al., "Transcriptional and posttranslational regulation of hyaluronan synthesis," FEBS J., VOL. 278 (9), PP. 1419-1428, 2011.

[9] S. Twarock et al., "Hyaluronan stabilizes focal adhesions, filopodia, and the proliferative phenotype in esophageal squamous carcinoma cells," J. Biol. Chem., VOL. 285 (30), PP. 23276-23284, 2010.

[10] B. P. Toole, "Hyaluronan: From extracellular glue to pericellular cue," Nat. Rev. Cancer, VOL. 4 (7), PP. 528-539, 2004.

[11] D. L. Russell and A. Salustri, "Extracellular matrix of the cumulus-oocyte complex," Semin. Reprod. Med., VOL. 24 (4), PP. 217-227, 2006.

[12] S. P. Evanko et al., "Adhesion and Migration," VOL. 31 (2), PP. 90-100, 2013.

[13] C. Tolg et al., "Hyaluronan and RHAMM in Wound Repair and the 'cancerization' of Stromal Tissues,” Biomed Res. Int., VOL. 2014,
(1), 2014.

[14] Y. Li et al., "Silencing of hyaluronan synthase 2 suppresses the malignant phenotype of invasive breast cancer cells," Int. J. Cancer, VOL. 120(12), PP. 2557-2567, 2007.

[15] Y. Li et al., "Hyaluronan synthase 2 regulates fibroblast senescence in pulmonary fibrosis," Matrix Biol., VOL. 55, PP. 35-48, 2017.

[16] H. Siiskonen et al., "Hyaluronan synthase 1: A mysterious enzyme with unexpected functions," Front. Immunol., VOL. 6, PP. 1-11, 2015.

[17] N. Itano et al., "Three isoforms of mammalian hyaluronan synthases have distinct enzymatic properties," J. Biol. Chem., VOL. 274 (35), PP. 25085-25092, 1999.

[18] T. D. Camenisch et al., "Disruption of hyaluronan synthase- 2 abrogates normal cardiac morphogenesis and hyaluronan-mediated transformation of epithelium to mesenchyme," VOL. 106 (3), PP. 1-12, 2000.

[19] J. A. Mack et al. "Enhanced inflammation and accelerated wound closure following tetraphorbol ester application or full-thickness wounding in mice lacking hyaluronan synthases Has1 and Has3 r," VOL. 5 (5), PP. 198-207, 2012.

[20] H. T. Mohamed et al., "Characterization of inflammatory breast cancer: a vibrational microspectroscopy and imaging approach at the cellular and tissue level," Analyst, VOL. 143 (24), PP. 6103-6112, 2018.

[21] A. Almond et al., "Hyaluronan: The Local Solution Conformation Determined by NMR and Computer Modeling is Close to a Contracted Left-handed 4-Fold Helix," J. Mol. Biol., VOL. 358 (5), PP. 1256-1269, 2006.

[22] P. Li et al., "Hyaluronan synthase 2 overexpression is correlated with the tumorigenesis and metastasis of human breast cancer," VOL. 8 (10), PP. 12101-12114, 2015.

[23] H. Urakawa et al., "Inhibition of hyaluronan synthesis in breast cancer cells by 4methylumbelliferone suppresses tumorigenicity in vitro and metastatic lesions of bone in vivo," Int. J. Cancer, VOL. 130 (2), PP. 454-466, 2012.

[24] B. Bernert et al., "Hyaluronan synthase 2 (HAS2) promotes breast cancer cell invasion by suppression of tissue metalloproteinase inhibitor 1 (TIMP-1)," J. Biol. Chem., VOL. 286 (49), PP. 42349-42359, 2011.

[25] T. Chanmee et al., "Excessive hyaluronan production promotes acquisition of cancer stem cell signatures through the coordinated regulation of twist and the transforming growth 
factor $\beta$ (TGF- $\beta$ )-snail signaling axis," J. Biol. Chem., VOL. 289 (38), PP. 26038-26056, 2014.

[26] P. Heldin et al., "Deregulation of hyaluronan synthesis, degradation and binding promotes breast cancer," J. Biochem., VOL. 154 (5), PP. 395-408, 2013.

[27] C. Laurich et al., "Hyaluronan mediates adhesion of metastatic colon carcinoma cells 1, J. Surg. Res., VOL. 122 (1), PP. 70-74, Nov. 2004.

[28] H. C. Lien et al., "Differential expression of hyaluronan synthase 2 in breast carcinoma and its biological significance," Histopathology,
VOL. 65 (3), PP. 328-339, 2014.

[29] Z. Zhang et al., "Hyaluronan synthase 2 expressed by cancer-associated fibroblasts promotes oral cancer invasion," J. Exp. Clin. Cancer Res., VOL. 35 (1), PP. 1-12, 2016.

[30] A. Jacobson et al., "Expression of human hyaluronan synthases in response to external stimuli.," Biochem. J., VOL. 348, (1), PP. 29-35, 2000.

[31] K. L. Schwertfeger et al., "Hyaluronan, inflammation, and breast cancer progression," Front. Immunol., VOL. 6 (6), PP. 1-12, 2015. 